\title{
The Crack Tip Fields for Anti-plane Crack in Functionally Graded Piezoelectric Materials with Arbitrarily Gradient Direction
}

\author{
Dai Yao ${ }^{1, a}$, Chong Xiao ${ }^{1, b}$, Sun Wei-hai ${ }^{1, a}$ \\ ${ }^{1}$ The Academy of Armored Force Engineering, Beijing, 100072 \\ adai_yao@sina.com, bchongxiao2005@163.com)
}

Keywords: crack tip fields, FGPMs, eigen-expansion method, anti-plane crack

\begin{abstract}
The higher order crack tip fields for anti-plane crack in functionally graded piezoelectric materials(FGPMs) under mechanical and electrical loadings are investigated. Although the elastic stiffness, piezoelectric parameter, and dielectric permittivity of FGPMs are assumed to be exponential functions, they are along arbitrary gradient direction. As usual, the crack surfaces are assumed to be electrically impermeable. Similar to the Williams' solution of homogeneous elastic materials, the high order crack tip stress and electric displacement fields are obtained by the method of eigen-expansion.
\end{abstract}

\section{Introduction}

To get rid of the possible interfacial failure, FGPMs have been introduced to reduce the stress concentration at the interface. In order to make FGPMs to have high strength, high reliability and long lifetime, the behaviors of cracks in brittle piezoelectric materials have to be analyzed. Therefore, fracture mechanics problems of a cracked body in FGPMs have received much attention in recent decades. Ma et al. [1] investigated the electro-elastic behavior of a Griffith's crack in a functionally graded piezoelectric strip. Ou [2] studied the internal crack problem located within one functionally graded piezoelectric strip. The crack is normal to the edge of the strip and the material properties vary along the direction of crack length. Hus and Chue [3] studied mode III arbitrarily oriented crack in an FGPM strip bonded to a homogeneous piezoelectric half plane. Yeh and Chue [4] investigated the anti-plane crack problem of a FGPM cracked strip bonded to an FGPM cracked half-plane. It should be mentioned that existing studies in fracture analysis of FGPMs are focused on the singular part of crack tip fields. No attempts have been made in giving the higher order crack tip fields for FGPMs with arbitrarily gradient direction. The purpose of this paper is to present the higher order crack tip fields and explicit expression of intensity factors.

\section{Basic equations}

Consider a crack in FGPMs under anti-plane shear tractions and in-plane electric displacements, as shown in Fig.1. The FGPM is poled in the $z$ direction and isotropic in the xoy plane. The present work employs the following exponential functions to describe the continuous variations of material properties,

$$
C_{44}=c_{440} e^{\beta_{1} x+\beta_{2} y}, \quad e_{15}=e_{150} e^{\beta_{1} x+\beta_{2} y}, \quad \varepsilon_{11}=\varepsilon_{110} e^{\beta_{1} x+\beta_{2} y}
$$

where $c_{440}$ is the shear modulus, $e_{150}$ is the piezoelectric coefficient, $\varepsilon_{110}$ is the dielectric parameter at $x=0, y=0$. 


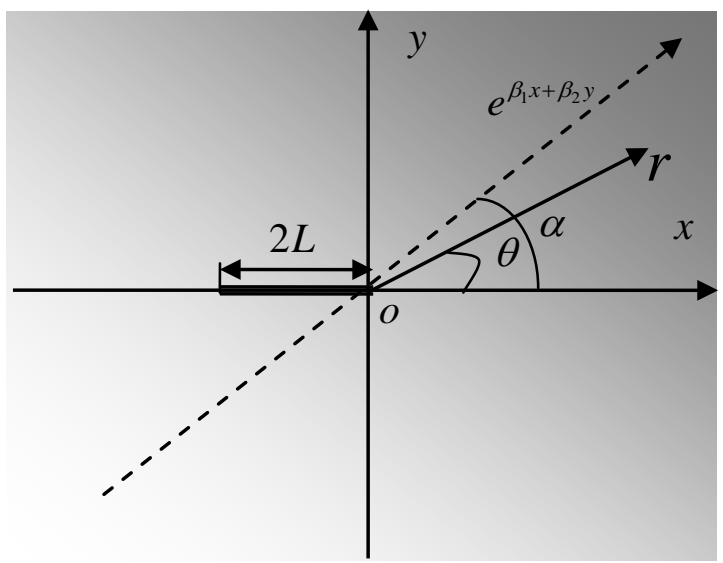

Fig.1Anti-plane crack in FGPMs

The governing equations can be written

$$
\begin{aligned}
& \nabla^{2} w+\beta\left[\cos (\theta-\alpha) \frac{\partial w}{\partial r}-\frac{1}{r} \sin (\theta-\alpha) \frac{\partial w}{\partial \theta}\right]=0 \\
& \nabla^{2} \phi+\beta\left[\left(\cos (\theta-\alpha) \frac{\partial \phi}{\partial r}-\frac{1}{r} \sin (\theta-\alpha) \frac{\partial \phi}{\partial \theta}\right)\right]=0
\end{aligned}
$$

where $\nabla^{2}=\frac{\partial^{2}}{\partial r^{2}}+\frac{1}{r} \frac{\partial}{\partial r}+\frac{1}{r^{2}} \frac{\partial^{2}}{\partial \theta^{2}}, \beta=\sqrt{\beta_{1}^{2}+\beta_{2}^{2}}$ and $\alpha=\arctan \left(\frac{\beta_{2}}{\beta_{1}}\right)$.

\section{The higher order crack-tip fields}

The displacement component $w$ and the electric potential $\phi$ can be expanded as

$$
w=\sum_{i=1}^{\infty} r^{\frac{i}{2}} w_{i}(\theta) \quad \phi=\sum_{i=1}^{\infty} r^{\frac{i}{2}} \phi_{i}(\theta)
$$

where $w_{n}(\theta)$ and $\phi_{n}(\theta)$ are eigen-functions.

Substitute Eq. (3) into Eq.(2) then yields the system of ordinary differential equations

$$
\left\{\begin{array}{l}
\frac{1}{4} \mathbf{w}_{1}(\theta)+\frac{\partial^{2} \mathbf{w}_{1}}{\partial \theta^{2}}=0 \\
\mathbf{w}_{2}(\theta)+\frac{\partial^{2} \mathbf{w}_{2}}{\partial \theta^{2}}=0 \\
\cdots \\
\frac{i^{2}}{4} \mathbf{w}_{i}+\frac{\partial^{2} \mathbf{w}_{i}}{\partial \theta^{2}}+\beta\left[\frac{i-2}{2} \cos (\theta-\alpha) \mathbf{w}_{i-2}-\sin (\theta-\alpha) \frac{\partial \mathbf{w}_{i-2}}{\partial \theta}\right]=0 \\
\cdots
\end{array}\right.
$$

according to the linear independence of $r^{-3 / 2}, r^{-1}, r^{-1 / 2}, \ldots, r^{i / 2-2}, \ldots$, where $\quad \mathbf{w}=\left[\begin{array}{l}w \\ \phi\end{array}\right]$.

In the case of electrically impermeable crack, the crack surfaces are free of electric charges and the electric displacement inside the crack is zero. As the crack surface is free, the boundary conditions are 


$$
\left.\sigma_{z y}\right|_{\theta= \pm \pi}=0,\left.D_{y}\right|_{\theta= \pm \pi}=0
$$

Furthermore, we have

$$
\frac{\partial \mathbf{w}_{i}}{\partial \theta}( \pm \pi)=0
$$

Solving the system of ordinary differential equations, we can obtain the results

$$
\left\{\begin{array}{l}
\mathbf{w}_{1}(\theta)=\mathbf{A}_{11} \sin \frac{\theta}{2} \\
\mathbf{w}_{2}(\theta)=\mathbf{A}_{21} \cos \theta \\
\mathbf{w}_{3}(\theta)=\mathbf{A}_{31} \sin \frac{3 \theta}{2}-\frac{\beta \mathbf{A}_{11}}{12}\left[\cos \frac{3 \theta}{2} \sin \alpha+3 \sin \left(\frac{\theta}{2}-\alpha\right)\right] \\
\mathbf{w}_{4}(\theta)=\mathbf{A}_{41} \cos 2 \theta-\frac{1}{4} \beta \mathbf{A}_{21} \cos \alpha \\
\mathbf{w}_{5}(\theta)=\mathbf{A}_{51} \sin \frac{5 \theta}{2}+\frac{\beta \mathbf{A}_{31}}{40}\left[\sin \left(\frac{5 \theta}{2}+\alpha\right)-\sin \left(\frac{5 \theta}{2}-\alpha\right)-10 \sin \left(\frac{\theta}{2}+2 \alpha\right)\right] \\
+\frac{\beta^{2} \mathbf{A}_{11}}{480}\left[15 \sin \frac{\theta}{2}+5 \sin \left(\frac{\theta}{2}+2 \alpha\right)-15 \sin \left(\frac{3 \theta}{2}-2 \alpha\right)-4 \sin \left(\frac{5 \theta}{2}-2 \alpha\right)+4 \sin \left(\frac{5 \theta}{2}+2 \alpha\right)\right] \\
\mathbf{w}_{6}(\theta)=\mathbf{A}_{61} \cos 3 \theta-\frac{\beta \mathbf{A}_{41}}{24}[\cos (3 \theta-\alpha)-\cos (3 \theta+\alpha)+6 \cos (\theta+\alpha)] \\
+\frac{\beta^{2} \mathbf{A}_{21}}{192}[\cos (3 \theta+2 \alpha)-\cos (3 \theta-2 \alpha)+6 \cos \theta+6 \cos (\theta-2 \alpha)] \\
\cdots . . .
\end{array}\right.
$$

where $\mathbf{A}_{i j}=\left[\begin{array}{l}A_{i j} \\ B_{i j}\end{array}\right]$ are the undetermined coefficients.

Substituting Eq. (7) into Eq.(3), the displacement component $w$ and the electric potential $\phi$ are obtained

$$
\begin{aligned}
& \mathbf{w}=r^{\frac{1}{2}} \mathbf{A}_{11} \sin \frac{\theta}{2}+r \mathbf{A}_{21} \cos \theta+r^{\frac{3}{2}}\left[\mathbf{A}_{31} \sin \frac{3 \theta}{2}-\frac{\beta \mathbf{A}_{11}}{12}\left(\cos \frac{3 \theta}{2} \sin \alpha+3 \sin \frac{\theta}{2}\right)\right]+ \\
& r^{2}\left(\mathbf{A}_{41} \cos 2 \theta-\frac{1}{4} \beta \mathbf{A}_{21} \cos \alpha\right)+r^{\frac{5}{2}}\left\{\left[\mathbf{A}_{51} \sin \frac{5 \theta}{2}+\frac{\beta \mathbf{A}_{31}}{40}\left[\sin \left(\frac{5 \theta}{2}+\alpha\right)-\sin \left(\frac{5 \theta}{2}-\alpha\right)-\right.\right.\right. \\
& \left.10 \sin \left(\frac{\theta}{2}+2 \alpha\right)\right]+\frac{\beta^{2} \mathbf{A}_{11}}{480}\left[15 \sin \frac{\theta}{2}+5 \sin \left(\frac{\theta}{2}+2 \alpha\right)-15 \sin \left(\frac{3 \theta}{2}-2 \alpha\right)-4 \sin \left(\frac{5 \theta}{2}-2 \alpha\right)+\right. \\
& \left.\left.4 \sin \left(\frac{5 \theta}{2}+2 \alpha\right)\right]\right\}+r^{3}\left\{\mathbf{A}_{61} \cos 3 \theta-\frac{\beta \mathbf{A}_{41}}{24}[\cos (3 \theta-\alpha)-\cos (3 \theta+\alpha)+6 \cos (\theta+\alpha)]+\right. \\
& \left.\frac{\beta^{2} \mathbf{A}_{21}}{192}[\cos (3 \theta+2 \alpha)-\cos (3 \theta-2 \alpha)+6 \cos \theta+6 \cos (\theta-2 \alpha)]\right\}+\cdots
\end{aligned}
$$

Then, the stress and the electric displacement components can be given by

$$
\begin{array}{ll}
\tau_{x z}=c_{44} w_{, x}+e_{15} \phi_{, x}, & \tau_{y z}=c_{44} w_{, y}+e_{15} \phi_{, y} \\
D_{x}=e_{15} w_{, x}-\varepsilon_{11} \phi_{, x}, & D_{y}=e_{15} w_{, y}-\varepsilon_{11} \phi_{, y}
\end{array}
$$

The mode III stress intensity factor (SIF) and electric displacement intensity factor (EDIF) of the crack tip are defined as 


$$
\left\{\begin{array}{l}
K^{T}=\lim _{r \rightarrow 0} \sqrt{2 \pi r} \sigma_{y z}(r, 0)=\frac{\sqrt{2 \pi}}{2}\left(c_{440} A_{11}-e_{150} B_{11}\right) \\
K^{D}=\lim _{r \rightarrow 0} \sqrt{2 \pi r} D_{y}(r, 0)=\frac{\sqrt{2 \pi}}{2}\left(e_{150} A_{11}+\varepsilon_{110} B_{11}\right)
\end{array}\right.
$$

\section{Conclusion}

The crack-tip higher order fields of FGPMs with arbitrarily gradient direction are obtained by the methods of eigen-expansion in this paper. The first two items of crack-tip higher order fields of FGPMs have the same mathematical forms as ones of homogeneous piezoelectric materials. The effect of non-homogeneity and gradient direction reflects only in the higher order items. Due to coupling effect of piezoelectric material, the stress intensity factor and electric displacement intensity factor are dependent on both displacement component and the electric potential. Obviously, the solutions provide the theoretical basis for experimental investigation and engineering application.

\section{Acknowledgements}

The research is supported by the National Natural Science Foundation of China (No11172332.)

\section{References}

[1] L. Ma, L.-Z. Wu, Z.-G. Zhou, L.-C. Guo: Composite Structures. Vol.69 (2005), p. 294

[2] Y.L. Ou, C.-H. Chue: International Journal of Solids and Structures. Vol.43 (2006), p. 6148

[3] W.H. Hsu, C.H. Chue: Meccanica. Vol.44 (2009), p. 519

[4] C.N. Yeh, C.H. Chue: Journal of the Chinese Institute of Engineers. Vol.35 (2012), p. 193 